Article

\title{
Risk-based mapping tools for surveillance and control of the invasive mosquito Aedes albopictus in Switzerland
}

\author{
Damiana Ravasi 1,*, Francesca Mangili ${ }^{2}$, David Huber ${ }^{2}$, Laura Azzimonti ${ }^{2}$, Lukas Engeler ${ }^{1}$, Nicola Vermes ${ }^{2}$, \\ Giacomo Del Rio ${ }^{2}$, Valeria Guidi ${ }^{1}$, Mauro Tonolla ${ }^{1}$ and Eleonora Flacio ${ }^{1}$ \\ 1 Institute of Microbiology (IM), Department for Environment Constructions and Design, University of Ap- \\ plied Sciences and Arts of Southern Switzerland (SUPSI), 6850 Mendrisio, Switzerland; dami- \\ ana.ravasi@supsi.ch (D.R.); lukas.engeler@supsi.ch (L.E.); valeria.guidi@supsi.ch (V.G.); mau- \\ ro.tonolla@supsi.ch (M.T.); eleonora.flacio@supsi.ch (E.F.) \\ 2 Dalle Molle Institute for Artificial Intelligence Studies (IDSIA), Department of Innovative Technologies, \\ University of Applied Sciences and Arts of Southern Switzerland (SUPSI), 6962 Lugano-Viganello, Switzer- \\ land; francesca.mangili@supsi.ch (F.M.); david.huber@supsi.ch (D.H.); laura.azzimonti@supsi.ch (L.A.); ni- \\ cola.vermes@gmail.com (N.V.); giacomo.delrio@supsi.ch (G.D.R.) \\ * Correspondence: damiana.ravasi@supsi.ch
}

\begin{abstract}
Background: In Switzerland, Aedes albopictus is well established in Ticino, south of the Alps, where surveillance and control are implemented. The mosquito has also been observed in Swiss cities north of the Alps. Decision-making tools are urgently needed by the local authorities in order to optimize surveillance and control. Methods: A regularized logistic regression was used to link the long-term dataset of Ae. albopictus occurrence in Ticino with socio-environmental predictors. The probability of establishment of Ae. albopictus was extrapolated to Switzerland and more finely to the cities of Basel and Zurich. Results: The model performed well, with an AUC of 0.86 . Ten socio-environmental predictors were selected as informative, including the road-based distance in minutes of travel by car from the nearest cell established in the previous year. The risk maps showed high suitability for Ae. albopictus establishment in the Central Plateau, the area of Basel and the lower Rhone Valley in the Canton of Valais. Conclusions: The areas identified as suitable for Ae. albopictus establishment are consistent with the actual current findings of tiger mosquito. Our approach provides a useful tool to prompt authorities' intervention in the areas where there is higher risk of introduction and establishment of Ae. albopictus.
\end{abstract}

Keywords: Aedes albopictus; ovitrap; regularized logistic regression; ecological niche model; environmental factors; surveillance

\section{Introduction}

In the last four decades, the Asian tiger mosquito, Aedes albopictus (Skuse, 1894), has been spreading globally outside its native range in Southeast Asia, to the point of being listed as one of the world's worst invasive alien species [1,2]. Its invasion success has been favoured by both extrinsic factors, such as increase of global trade and travel, climate change and lack of efficient control, and intrinsic factors, such as strong physiological and ecological plasticity [1,3,4]. For instance, the ability to produce cold-tolerant eggs through a photoperiodic diapause response allows the mosquito to overwinter at the egg stage in temperate climates $[5,6]$.

Thanks to its adaptation to use artificial containers as breeding sites, Ae. albopictus thrives in suburban and urban environments [1]. Host-seeking adult females can cause severe nuisance due to their aggressive daytime outdoor biting activity. In addition, their ability to transmit several arboviruses, including dengue, chikungunya, Zika and yellow fever viruses, raises high public concern $[7,8]$. The increase of Aedes-borne diseases in Europe is becoming quite alarming with a succession of epidemics of dengue and 
chikungunya in different countries since the years 2000 [9]. Rising temperatures due to climate change may exacerbate the risk of epidemics by allowing increased mosquito survival, reproduction and biting rate, faster viral amplification and longer transmission season [10].

In Europe, Ae. albopictus appeared for the first time in Italy in the 1990s and then expanded to most areas of the country and to other countries passively through the various human transportation networks (Mosquito Maps, http://ecdc.europa.eu/). In Switzerland, its presence was recorded for the first time in 2003 in the southern side of the Alps, in the Canton of Ticino (hereafter referred to as Ticino), at a service area on the European route E35, close to the Italian border [11,12]. Despite containment measures being implemented since its first appearance, Ae. albopictus gradually formed permanent overwintering populations and expanded northwards across the lower valleys [13,14]. Today it is considered well established (with reproduction and overwintering) in most urban areas of Ticino.

The spread of Ae. albopictus in Ticino has been actively monitored since 2003 by the cantonal Working Group for Mosquitoes (Gruppo Lavoro Zanzare, GLZ) through an extensive surveillance system based on the deployment of oviposition traps (ovitraps) for collection and counting of Aedes eggs. Ovitrap data indicate suitability of a habitat for oviposition and therefore for the establishment of populations. The surveillance has gradually expanded over the years to cover larger areas in response to reported and suspected presence of the mosquito [13]. During the first phases of Ae. albopictus invasion in 2003, the surveillance focused on suspected entry points near the border with Italy, such as motorway service areas, high traffic areas, large parking lots, import shops and goods handling stations. Between 2004 and 2008, as the mosquito started to spread and to become established at several sites, the surveillance was widened to include industrial areas, larger car parks and public areas in affected municipalities. From 2009, as the mosquito started to colonize urban areas, the monitoring was extended to an area-wide surveillance network covering the urban areas of entire municipalities, with the adoption of a grid system of $250 \mathrm{~m} \times 250 \mathrm{~m}$ units to standardize the distribution of ovitraps on the territory. Currently, more than 80 municipalities are involved in the surveillance and control of the mosquito, covering more than $90 \%$ of the total human population of Ticino.

The introduction and spread of Ae. albopictus is also monitored since 2013 at the Swiss national level, along the main potential dispersal routes (i.e., highways, airports, river ports and freight stations) [15]. To date, Ae. albopictus has been observed in the Canton of Grisons, south of the Alps, and is considered established in the southern part of the canton. North of the Alps, the mosquito has become firmly established since 2018 in an area of Basel adjacent to the motorway toll on the Swiss-French border [16]. Small populations of Ae. albopictus were also recorded recently in two areas of Zurich: an international bus station located in the centre of the city, near the main train station, and a suburban neighbourhood in the Wollishofen district (Swiss Mosquito Network, http://www.mosquitoes-switzerland.ch). Possible foci of introduction have also been observed in the central (e.g., Cantons of Uri and Luzern), northern (e.g., Cantons of Solothurn, Aargau, Basel-Landt and Basel-Stadt), northeastern (e.g., Cantons of Zurich and Schaffhausen) and western (e.g., Rhone Valley in the Canton of Valais and Cantons of Fribourg, Vaud and Geneva) parts of Switzerland (Swiss Mosquito Network). These observations, along with the concurrent rapid spread in other European countries, suggest that the tiger mosquito will colonize more and more areas in Switzerland in the near future, introducing the risk of local arbovirus transmission following imported human cases.

Since extensive surveillance might not be economically viable in many municipalities, the early identification of the most suitable areas for spread and establishment of $A e$. albopictus becomes a critical tool to support the authorities in vector management planning. Ecological niche models can be used to evaluate the potential risk of spread and establishment of invasive species [17]. In particular, correlative distribution models 
identify relationships between species' presence-only or combined presence-absence data and socio environmental variables (e.g., meteorological data, habitat type classes and host population density estimates) and use these relationships to make predictions on the potential distribution of the species in unsampled areas of the study region [18]. Temperatures and precipitations have a strong impact on the development and survival of the different Ae. albopictus stages [19,20]. Therefore, many models have focused on meteorological variables to predict areas suitable for colonization [4,21]. In Switzerland, remotely sensed land surface temperature data were used by Neteler and colleagues [22] to identify suitable areas for adult survival and overwintering of diapausing eggs and to project climate change induced range shifts of Ae. albopictus.

In addition to weather conditions, the distribution Ae. albopictus can also be influenced by other aspects, such as environmental features (e.g., elevation, type of habitat and vegetation canopy) and anthropogenic factors (e.g., human population density, transport network and travel) reflecting the impact of human movements and urbanization on the spread of the mosquito [23,24]. In this context, modelling approaches such as machine learning represent powerful tools as they provide the required flexibility to handle complex and multiple interacting elements. Many algorithms have been used to predict the distribution of mosquito species at different spatial scales, from regional $[25,26]$, to national $[27,28]$, continental $[3,4,23]$ and global $[29,30]$, with different outcomes on the most informative predictors.

Here we used the long-term dataset of Ae. albopictus presence-absence records in Ticino to train and validate a regularized logistic regression and to forecast the probability of establishment of Ae. albopictus. The most informative explanatory features for the prediction of establishment of Ae. albopictus in Ticino were identified. The model was then used to extrapolate the probability of establishment in the whole Switzerland, for which there are almost no establishment records, and in the cities of Basel and Zurich, where only a small number of occurrences is available for analysis. We created risk maps of $A e$. albopictus establishment with the goal to help cantonal and municipal authorities in optimizing efforts and resources by targeting vector surveillance and control in the areas where Ae. albopictus has not yet been recorded but where the habitat appears to be suitable for establishment during summer months if mosquitoes are introduced.

\section{Materials and Methods}

\subsection{Study area}

The study area comprises the entire $41,285 \mathrm{~km}^{2}$ territory of Switzerland ( $45^{\circ}$ to $48^{\circ} \mathrm{N}$ and $5^{\circ}$ to $\left.11^{\circ} \mathrm{E}\right)$. Switzerland lies in the heart of Europe and is mostly covered by agricultural land (40\%), forest and woodland (30\%), settlement areas (7.5\%) and bodies of water $(4 \%)$. As a result of its geographical position and its complex topography, the country has a varied climate. The Alps form an important climatic barrier between the north and south of Switzerland. In the Central Plateau, which lies north of the Alps, between the Swiss Jura and the Swiss Alps, the climate is moderately continental, with cold winters often reaching freezing temperatures in January, and warm summers. In the south of the Alps, namely in Ticino, the climate is strongly affected by the Mediterranean Sea, with mild winters and summers warm and humid, and sometimes hot. Switzerland is particularly affected by climate change. Since measurements began in 1864 , the average temperature has already increased by approximately $2^{\circ} \mathrm{C}$. This is about twice the average global temperature rise. The five warmest years in the measurement series 1864-2019 were all recorded after 2010. Other evidence of climate change are heatwaves, more hot days and nights, and shrinking snow cover on the Central Plateau. The frost days have decreased of up to $60 \%$ since 1961 . Heavy precipitation events have also become more intense and more frequent [31].

The overall area of Switzerland was rasterized into 1,465,516 square pixels (or cells) with a 200-m resolution based on the freely available digital height model of Switzerland DHM25 / 200m with coordinate system CH1903 / LV03 (EPSG: 21781) LN02 (EPSG: 5728), 
produced by the Swiss Federal Office of Topography [32]. The digital height model DHM25 / 200m is a dataset representing the three-dimensional form of the earth's surface without vegetation or built-up areas. All data layers used in the model were matched to this grid.

\subsection{Response variable}

Data for Ae. albopictus in Ticino are available through the long-term surveillance system based on the deployment of ovitraps [13,33]. The number of ovitraps has increased during the years, going from 57 traps in 2004 to a maximum of 1,389 traps deployed in 2013. The ovitraps are deployed at the beginning of the active season of the mosquito, in May, and are inspected every two weeks until the end of September. At each inspection round, the wooden slat of each ovitrap, where mosquitoes lay their eggs, is collected and replaced with a new one. In the laboratory, the slats are analysed under a stereo microscope (EZ4 D, Leica Microsystems, Germany) and Ae. albopictus eggs are counted. Since 2013, although the wooden slats are still replaced every two weeks, the regular biweekly analysis has been maintained only for one municipality per district, while for the rest of the municipalities the analysis is performed only every second inspection round. Data from each ovitrap, including geographic coordinates, date of collection and number of Ae. albopictus eggs, is stored in a national database managed by the Institute of microbiology and the info fauna - Swiss Centre for Cartography of the Fauna (http://www.cscf.ch/).

We used ovitrap data from 2004 to 2015. As of 2015, two new mosquito species, namely Ae. koreicus and Ae. japonicus are present in different areas of Ticino. Since the eggs of these two species cannot be morphologically differentiated from the eggs of $A e$. albopictus without resorting to special microscopy equipment and expertise, data collected after 2015 were not considered for this study.

Since the wooden slats are deployed in the field for periods of two weeks, the yearly surveillance period was divided in ten periods of two weeks (from period 1 including calendar weeks 20-21 to period 10 including calendar weeks 38-39). In order to assess the stable presence of Ae. albopictus in an ovitrap each year, we defined an establishment indicator as follows: we say that establishment for an ovitrap occurred when a sequence of consecutive positive wooden slats was observed with the last positive slat occurring more than 28 days after the first one, therefore accounting for the fact that from 2013 slats were analysed at alternate rounds [14]. Subsequently, each ovitrap was assigned, based on its geographical position, to the appropriate $200 \mathrm{~m} \times 200 \mathrm{~m}$ cell of the model grid. Aedes albopictus was considered established in a cell in a given year if there was establishment in at least one of the ovitraps located in the cell. Thus, the response variable Established was defined as 1 (TRUE) if there had been establishment in a cell in a given year, 0 otherwise. This resulted in a total number of 7,268 establishment / non-establishment data (Table 1). Figure 1 shows the occurrence of Ae. albopictus from 2005 to 2015.

Table 1. Number of ovitraps analysed in Ticino for the presence of Ae. albopictus, ovitraps with establishment of the mosquito, cells observed and cells with establishment.

\begin{tabular}{ccccccccccccc}
\hline & $\mathbf{2 0 0 4}$ & $\mathbf{2 0 0 5}$ & $\mathbf{2 0 0 6}$ & $\mathbf{2 0 0 7}$ & $\mathbf{2 0 0 8}$ & $\mathbf{2 0 0 9}$ & $\mathbf{2 0 1 0}$ & $\mathbf{2 0 1 1}$ & $\mathbf{2 0 1 2}$ & $\mathbf{2 0 1 3}$ & $\mathbf{2 0 1 4}$ & $\mathbf{2 0 1 5}$ \\
\hline Ovitraps analysed & 57 & 189 & 235 & 292 & 466 & 1,241 & 1,342 & 1,357 & 1,361 & 1,389 & 1,022 & 1,031 \\
Ovitraps established & 3 & 0 & 5 & 23 & 94 & 144 & 181 & 265 & 580 & 524 & 463 & 793 \\
Cells observed & 25 & 76 & 82 & 101 & 163 & 887 & 973 & 983 & 1,017 & 1,108 & 925 & 928 \\
Cells established & 3 & 0 & 3 & 16 & 44 & 106 & 144 & 211 & 466 & 456 & 425 & 727 \\
\hline
\end{tabular}

Established data for years 2005 to 2012 were used to train the model. Data from 2004 were not used in the model but were used, as described later, to calculate one of the predictors (namely carD), which requires establishment data from the previous year to be 
computed. The training dataset of 4,282 observed cells was composed of 990 established data and 3,292 non-established data. The model validation was performed with 2,961 cells for years 2013 to 2015 comprising 1,608 established data and 1,353 non-established data (Table 1).

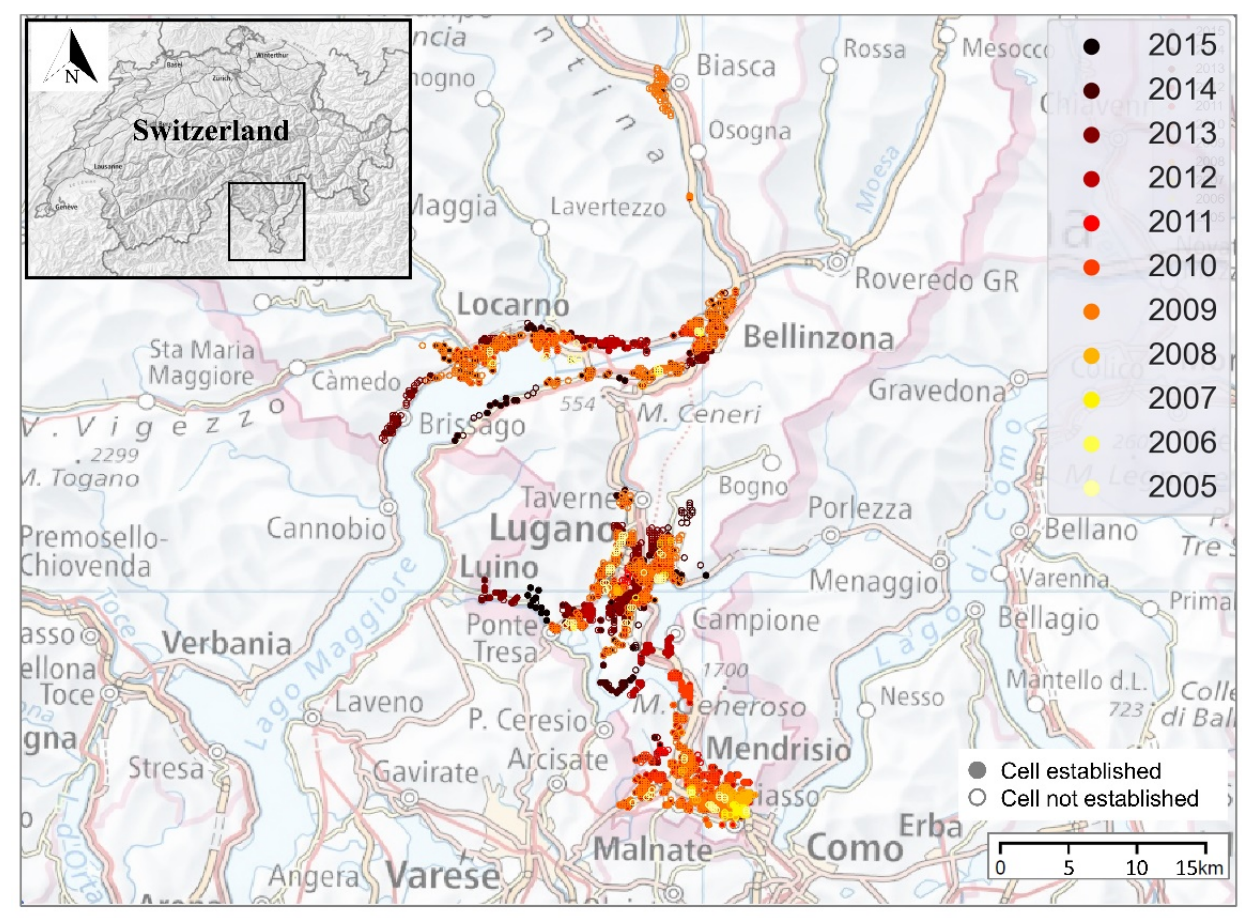

Figure 1. Occurrence of Ae. albopictus in Ticino, Switzerland, from 2005 to 2015. Each dot is an observed cell. Solid dots represent cells with establishment of the mosquito, empty dot represent cells without establishment. Maps modified from https://map.geoadmin.ch/.

\subsection{Predictors used for Ae. albopictus habitat suitability}

A total of 79 candidate predictors were selected based on their potential relevance to Ae. albopictus distribution (Table S1). These included static attributes for cell terrain morphology $(n=3)$, type of land cover $(n=32)$ and permanent host population $(n=1)$, and dynamic features such as meteorological variables $(n=42)$ and travel distance from the nearest cell with establishment $(n=1)$.

Meteorological predictors were derived from the MeteoSwiss spatial climate daily datasets (source: MeteoSwiss). The temperature datasets are constructed through interpolation of daily minimum, maximum and mean temperatures from a weather station network of approximately 90 weather stations (measuring free-air temperature 2 $\mathrm{m}$ above ground level) to a 1-km resolution grid in the Swiss coordinate system CH1903 $[34,35]$. The precipitation dataset is built from daily precipitation totals measured at the MeteoSwiss high-resolution rain-gauge network [36].

The daily datasets were processed with Apache Spark ${ }^{\mathrm{TM}} 2.44$ [37] to compute seasonal predictors. Cold season (c) for a given year $y$ included the months from the beginning of December of the previous year $(y-1)$ to the end of February. Warm season $(w)$ lasted from the beginning of May to the end of September. We calculated the seasonal average (avg) and the percentiles 5, 25, 75 and 95 (p5, p25, p75 and p95) for the following data series: daily minimum, maximum and mean temperatures (Tmin, Tmax, Tmean) and daily rainfall (RAIN). Percentiles 5 and 25 were not used for RAIN (both warm and 
cold seasons) as they are constantly equal to zero. In total, we obtained 36 meteorological seasonal predictors.

To account for meteorological conditions on a more refined temporal scale, we also included some features based on two-week statistics of MeteoSwiss data series. These features account better for prolonged extreme meteorological conditions than seasonal predictors. Namely, minMmaxTmin and minMavgTmin are defined as the minima of the maximum and average values of Tmin over any two-week period of the year and are introduced to measure how cold were two of the coldest two-week periods of each year; features maxMminTmax and maxMavgTmax are defined as the maxima of the minimum and average values of Tmax over any two-week period of the year and are introduced to measure how hot were two of the hottest two-week periods of each year; maxMavgRAIN and minMavgRAIN are defined as the minimum and the maximum of the average values of RAIN over any two-week period of the warm season and are introduced to measure the amount of rainfall in one of the, respectively, wettest and driest periods of the warm season. The reader is referred to Appendix A for a more detailed description of these features.

Since the coordinate system of the MeteoSwiss grid matched the one of the digital height model DHM25 / 200m, except for the lower resolution, the meteorological predictors' layer was overlapped to the DHM25 / $200 \mathrm{~m}$.

Three terrain morphology features were derived from the digital height model of Switzerland DHM25 / 200m: cell elevation, aspect (i.e., land orientation) and slope. These topographic features give indirect measures of the climate at the cell's scale and can influence the biotic conditions of mosquito and the choice of suitable breeding sites.

The way a habitat type is distributed relates directly to habitat preferences in mosquitoes. In Switzerland, there are 32 types of land cover, such as artificial surfaces, agricultural areas, forests, semi-natural areas, wetlands and water bodies (Table S1). Land cover data were obtained from CORINE Land Cover 2012 (CLC-CH 2012), where images acquired by earth observation satellites in 2011-2012 were used as the main source data to derive land cover classes (@) European Union, Copernicus Land Monitoring Service 2012, European Environment Agency; [38]). Percentages of coverage for each class were extracted for each $200 \mathrm{~m} \times 200 \mathrm{~m}$ cell.

The total human host population was extrapolated from the layer STATPOP2016 (Swiss Federal Statistical Office, GEOSTAT), based on 2016 statistical data of the total permanent human resident population. The punctual data of the STATPOP2016 $100 \times 100$ $\mathrm{m}$ grid were forced on the reference $200 \mathrm{~m} \times 200 \mathrm{~m}$ grid and the total population in each cell was calculated.

In order to account for the propagation paths of adult mosquitoes in neighbourhood areas, we also included in the set of predictors the distance of each cell from the closest cell in which mosquitoes had established the year before. As mosquitoes can be displaced by means of passive transport (especially cars) in their adult forms [39], distance was measured by the travel time in minutes necessary to go from the centre of one cell to the centre of the other. For each cell $c$ at year $y$, the travel time from all cells established at year $y-1$ to cell $c$ was computed and the minimum value obtained was retained as the value of the feature carD, thus estimating the minimum duration of a passive transport of mosquitoes to cell $c$. The duration of car travels was estimated using the Open Source Routing Machine (OSRM, http://project-osrm.org/, [40]). OSRM is a free C++ implementation of a high-performance routing engine for shortest paths in road networks combining routing algorithms with the open and free road network data of the OpenStreetMap project (OSM, https://www.openstreetmap.org, [41]). Notice that the carD feature for the years 2005 to 2015 is based on the values of feature Established (response variable) of years 2004 to 2014 .

\subsection{Predictive model}


Established and non-established data were modelled using an ensemble of regularized logistic regression models to predict Ae. albopictus establishment probability. By aggregating multiple different models, the ensemble approach tries to compensate the weaknesses of individual models to produce a more robust prediction [42]. The ensemble included eight models learned on different training datasets using the Logistic Regression methods included in the scikit-learn Python package 0.24 [43]. Logistic regression assigns a weight to each predictor and returns the probability relative to the class Established. Most of the predictors considered were correlated and, therefore, redundant. For this reason, we adopted a regression approach with a strong penalization: the Least $\mathrm{Ab}$ solute Shrinkage and Selection Operator (LASSO) [44] was used for predictors' selection and regularization to avoid parameter overfitting. Lasso is a penalized least square method that shrinks the coefficient space by imposing an L1 penalty (sum of absolute values) on the regression coefficients. This means that the predictors' selection was done automatically, based on a regularization parameter $C$ representing the strength of the penalization (higher values of $C$ implying a smaller number of selected predictors). Different values of $C$ were assessed using a cross-validation over the individual models of the ensemble as described below. We noticed that, inside the range $[0.01,1]$ the choice of $C$ was not significantly affecting the performance. We therefore chose the intermediate value of 0.1 , which produced models selecting between nine and 14 most relevant features.

The eight models of the ensemble were obtained as follows. Ticino data from 2005 to 2012 were divided into eight folds and each model was trained using only seven folds, using a leave-one-year-out cross-validation strategy. Therefore, rather than randomly splitting the data into $k$ folds, we grouped them by year to increase the diversity between models. We thus obtained eight models, each one learned on a subset of the training data obtained by leaving out a different year. Finally, to improve the overall predictive performance and provide an estimate of the prediction uncertainty, we used the eight models within an ensemble approach producing a single ensemble prediction and a measure of the prediction uncertainty, respectively, as the mean and the standard deviation of the eight models' predictions.

The performance of the ensemble model was evaluated using the area under the receiver operating curve (AUC). The AUC represents the probability that, given two random cells, one established and one non-established, the classifier assigns a larger risk of establishment to the established cell. This gives us a measure of how good the classifier is in ordering the suitability of different areas to Ae. albopictus establishment. In a first cross-validation assessment, we computed the AUC of each individual model of the ensemble on the left-out year of the training dataset. Models leaving out years 2005 and 2006 were excluded due to lack or very small number of established cells in those two years. This cross-validation strategy, besides producing more diverse models, avoids overestimating the performance by excluding data from the same year as the test set from the training set. In a second assessment, we compared the prediction of the probability of establishment (hereafter also referred to as risk of establishment) of Ae. albopictus in Ticino based on the predictor features of the years 2013, 2014 and 2015 to the actual data of establishment for those years, both for each individual model and for the ensemble prediction.

Due to the Lasso penalization adopted, the eight trained models selected a subset of the initial features by assigning a null weight to the features that granted no improvement to the outcome of the prediction. This allowed us to identify the most informative explanatory features for the prediction of the distribution of Ae. albopictus in Ticino by looking at the proportion of individual models of the ensemble assigning them a non-zero coefficient.

The ensemble model was then used to create maps estimating the risk of Ae. albopictus establishment in Switzerland. For each $200 \mathrm{~m}$ x $200 \mathrm{~m}$ cell of the whole Switzerland, we computed the predictive features and input them to the model to predict the 
probability of establishment. Since data about establishment in the past were available only for Ticino, it was not possible to compute the carD feature in the rest of the country. Therefore, we kept a fixed carD of 0.5 minutes (30 seconds) for all cells, implying that establishment has already occurred in the neighbourhood. This provided an overview of the areas in Switzerland which are mostly at risk in case a first colony of mosquitoes arrives.

We then focused on two areas, the cities of Basel and Zurich, for which we did have records of positive ovitraps for 2019 (37 points of first establishment for Basel and 16 for Zurich; data kindly provided by the Inspection body for chemical and biosafety (KCB) of the Cantonal Laboratory of Basel-Stadt, the Swiss Tropical and Public Health Institute, the Urban Pest Advisory Service of the City of Zurich and the Office for Waste, Water, Energy and Air (AWEL) of the Canton of Zurich). In this case, it was possible to build the carD feature necessary to build the risk maps by computing the smallest distance between each cell of Basel or Zurich and the identified position of 2019 establishments.

In order to build suitability maps of Ae. albopictus establishment, it was also necessary to create scenarios for the meteorological conditions of the coming years. For this, we decided to use meteorological data from years 2015, 2016, 2017 and 2018 in order to analyse multiple realistic scenarios. We finally produced two types of maps, by projecting probabilities of establishment onto Swiss national maps (Federal Office of Topography swisstopo): a suitability map showing the average of the 32 establishment probabilities predicted by the eight models of the ensemble for the four meteorological scenarios (years 2015 to 2018) and an uncertainty map representing the standard deviation of the 32 predictions, which provides an indication about the reliability of risk estimate displayed in the suitability map.

\section{Results}

\subsection{Informative predictors}

The importance of the predictors was evaluated based on the coefficients assigned to them by each of the eight Lasso logistic regression models trained in cross-validation on years 2005 to 2012. Table 2 shows the predictors that were assigned a non-null coefficient by at least half of the models of the ensemble and the sign of the assigned coefficients. Each feature is ranked based on its frequency, i.e., the proportion of models using it as a predictor of the establishment probability. The more models select a predictor, the more likely it is that it provides useful information about mosquitoes' establishment. The sign of the coefficients indicates the direction of the relation. For instance, the negative sign of the coefficient associated to elevation means that the higher the elevation of a $200 \mathrm{~m} \times 200$ m cell, the lower the probability of Ae. albopictus establishment (Table 2).

Table 2. Informative predictors selected by lasso regularization.

\begin{tabular}{cccc}
\hline Predictor & Description & Frequency $^{1}$ & Sign $^{2}$ \\
\hline elevation & Altitude in m.a.s.l. & 1.000 & -1.0 \\
wTmin_p5 & $5^{\text {th }}$ percentile of the minimum temperature in the warm season & 1.000 & -1.0 \\
wRAIN_avg & Average of precipitations in the warm season & 1.000 & -1.0 \\
cTmax_p25 & 25 $^{\text {th }}$ percentile of the maximum temperature in the cold season & 1.000 & 1.0 \\
carD & Road-based distance in minutes of travel by car from the nearest & 1.000 & -1.0 \\
& cell established in the previous year & 1.000 & -1.0 \\
clc121 & Percentage of industrial or commercial units covering a cell & 1.0 \\
minMavgTmin & Average daily minimum temperature (Tmin) observed during & 0.875 & -1.0 \\
& the two-week period of the year with the lowest average Tmin & \\
minMavgRAIN & Average precipitation observed during the two-week period of & 0.875 & 1.0
\end{tabular}


the warm season with the lowest average precipitation

cRAIN_p75 $\quad 75^{\text {th }}$ percentile of the precipitations in the cold season $\quad 0.750 \quad 0.500$ wTmax_p75 $\quad 75^{\text {th }}$ percentile of the maximum temperature in the warm season $\quad 0.500 \quad 1.0$

${ }^{1}$ Frequency: fraction of individual models of the ensemble including the feature.

${ }^{2}$ Sign: sign of the feature coefficient.

Amongst all 79 candidate predictors, only ten were considered informative by at least half of the eight cross-validation models, and thus most significant for predicting the probability of Ae. albopictus establishment in Ticino. Figure 2 shows the values of the coefficients assigned to these features by the eight models. It can be noticed that coefficient values are consistent across models. Six of the ten predictors showed a negative relation to the probability of establishment: elevation, the fifth percentile of the minimum temperature in the warm season (wTmin_p5), the average of precipitations in the warm season (wRAIN_avg), the road-based distance in minutes of travel by car from the nearest cell established in the previous year (carD), the percentage of industrial or commercial units covering a cell (clc121) and average daily minimum temperature (Tmin) observed during the two-week period of the year with the lowest average of Tmin (minMavgTmin). On the contrary, the $25^{\text {th }}$ percentile of the maximum temperature in the cold season (cTmax_p25), the average precipitation observed during the two-week period of the warm season with the lowest average precipitations (minMavgRAIN), the $75^{\text {th }}$ percentile of the precipitations in the cold season (cRAIN_75) and the $75^{\text {th }}$ percentile of the maximum temperature in the warm season (wTmax_p75) showed a positive relation to the probability of establishment.

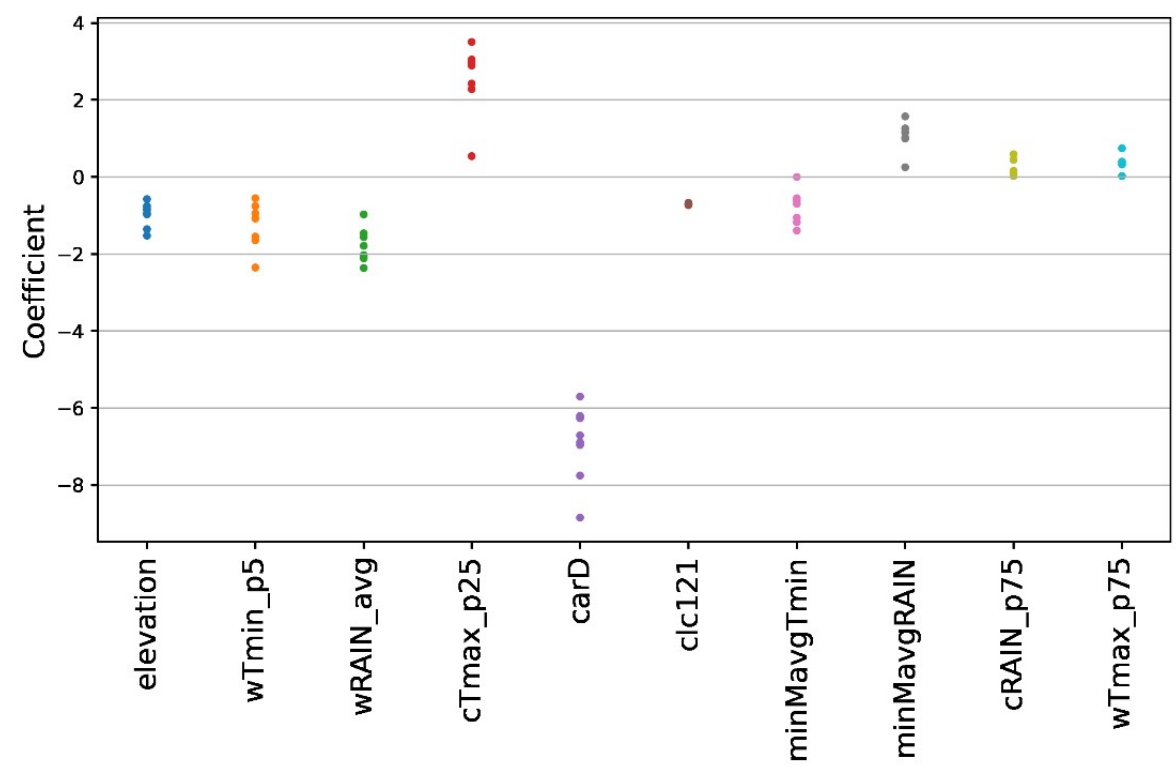

Figure 2. Coefficients assigned by the ensemble models to the mostly used features (i.e., features used by at least half of the models).

\subsection{Model outputs}

The performance of the ensemble was firstly assessed computing the average and standard deviation of the AUC of six individual models of the ensemble on the left-out year of the training dataset. Models leaving out years 2005 and 2006 were excluded due to lack or very small number of established cells in those two years. Secondly, to better validate the ensemble model, the AUC of each individual model was assessed on the es- 
tablishment data of years 2013, 2014 and 2015 separately. The cross-validation yearly means and standard deviations of the AUCs obtained by the individual models of the ensemble are given in the first row of Table 3. The second row gives, instead, the AUC of the ensemble prediction, i.e., first the individual model predictions are averaged, then the AUC of the average prediction is computed (not meaningful in the case of cross-validation). We observe that the AUC obtained by the cross-validation over years 2007 to 2012 (i.e., 0.856) is better than that obtained on the more recent years used for testing (years 2013 to 2015, AUCs from 0.670 to 0.748) when the establishment was more widespread.

Table 3. Prediction performance of the regularized logistic regression in cross-validation and on the test dataset.

\begin{tabular}{lcccc}
\hline & $\begin{array}{c}\text { Cross Valida- } \\
\text { tion }\end{array}$ \\
& $\begin{array}{c}\text { (2007-2012) } \\
\text { (2013 }\end{array}$ & $\mathbf{2 0 1 4}$ & $\mathbf{2 0 1 5}$ \\
\hline $\begin{array}{l}\text { Mean and standard deviation of the AUCs } \\
\text { of the individual models of the ensemble }\end{array}$ & $0.856(0.047)$ & $0.670(0.063)$ & $0.731(0.008)$ & $0.740(0.007)$ \\
AUC of the ensemble average prediction & & 0.748 & 0.733 & 0.741 \\
\hline
\end{tabular}

${ }^{1}$ Cross-validation considers only the six models for which the left-out-year used for validation has enough established cells (namely, years 2007 to 2012).

${ }^{2}$ Standard deviations (SDs) are presented in parentheses.

The ensemble model was used to predict the probability of Ae. albopictus establishment in the whole Switzerland and in the cities of Basel and Zurich for each of the four scenarios represented by the meteorological conditions observed in years 2015, 2016, 2017 and 2018. We thus obtained 32 estimates of the probability of establishment generated by the eight individual models of the ensemble over the four climate conditions considered for each $200 \mathrm{~m} \times 200 \mathrm{~m}$ cell of the areas of interest. Using such predictions, we generated five risk maps for each area: four yearly risk maps with the average of the 8 risk estimates obtained for each of the four years and a summary risk map, showing the overall average of the 32 risk estimates (Figures 3, 4 and 5, left column). Each risk map is accompanied by an uncertainty map showing the standard deviation of the predictions used in the corresponding map (Figures 3, 4 and 5, right column) normalized between 0 and the maximum observed value of standard deviation over all maps (namely, 0.31). Uncertainty maps describe the reliability of the prediction, higher values of the standard deviation meaning that the prediction is less reliable. We notice for instance a red spot in the northeaster part of Switzerland in the 2015 standard deviation map of Figure 3, meaning that, for the peculiar meteorological conditions of 2015, the risk estimated in that area nearby lake Constance has a large variability and, therefore, should not be trusted. 


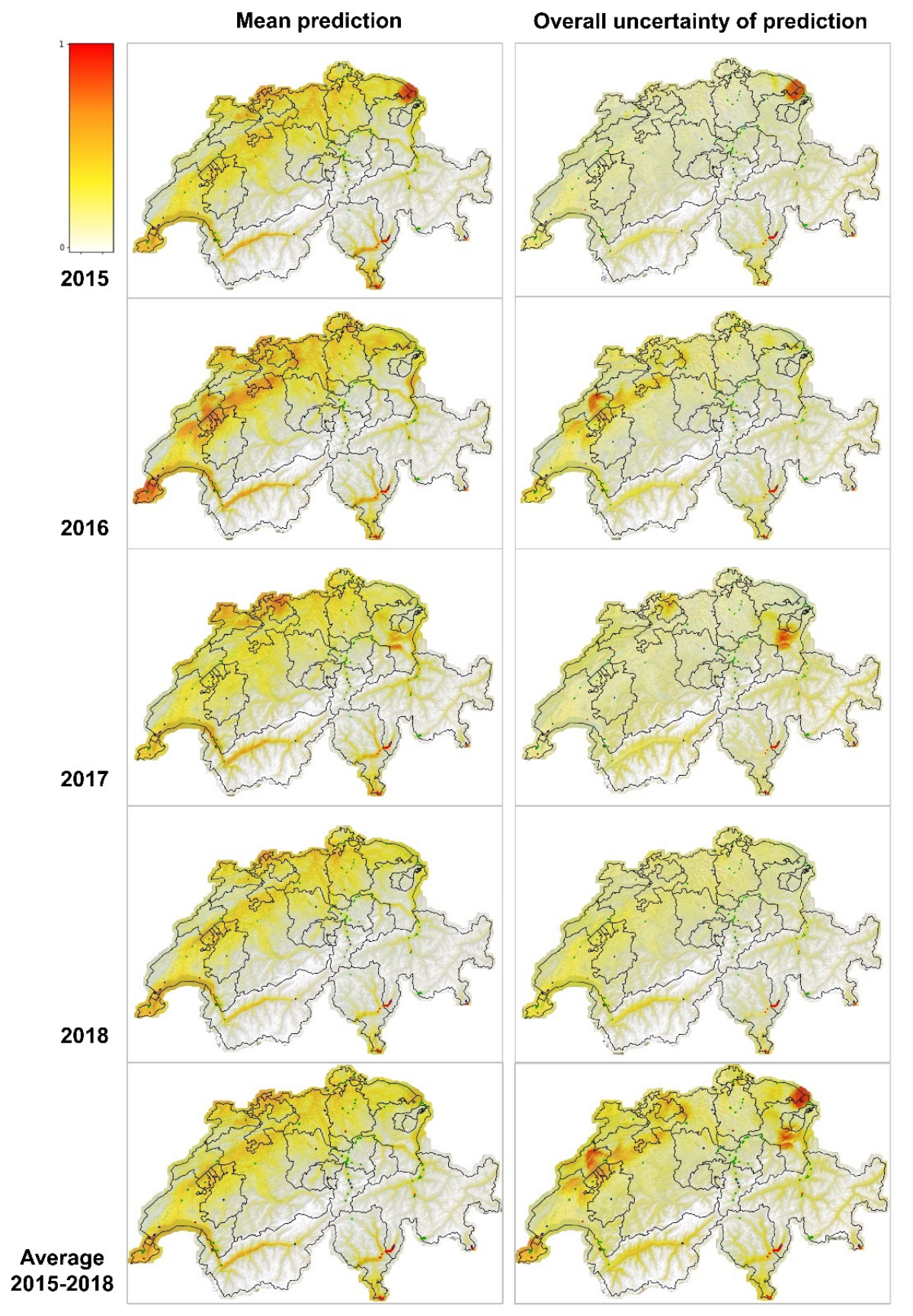

Figure 3. Maps of probabilities of establishment for Switzerland predicted by the regularized logistic regression for 2015 to 2018. The maps in the left column correspond to the risk estimates based on the meteorological conditions of each individual year (maps named 2015, 2016, 2017, 2018) and the average risk over the four years (map named average 2015-2018). Those in the right column represent the uncertainty of the corresponding predictions (higher values representing more uncertain predictions). The colour gradient shows the probability of establishment or the normalized standard deviation from 0 (white) to 1 (red). Dots show cells where the presence of $A e$. albopictus was monitored in 2021, the colour green representing cases where it was found absent from the cell, blue where it was present but not established and red where it was established (data 
source: Swiss Mosquito Network and info fauna - Swiss Centre for Cartography of the Fauna). Maps modified from https://map.geoadmin.ch/.

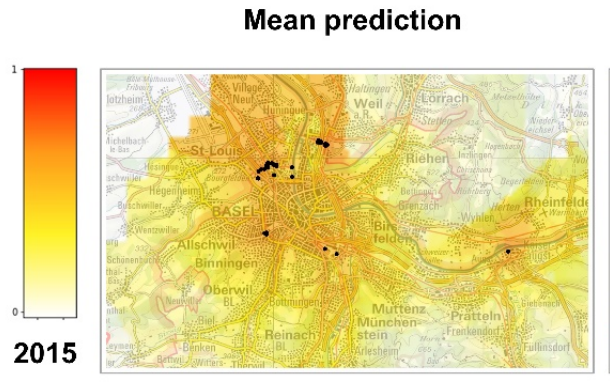

\section{Overall uncertainty of prediction}

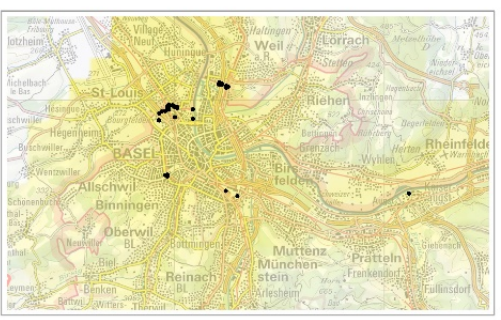

2016
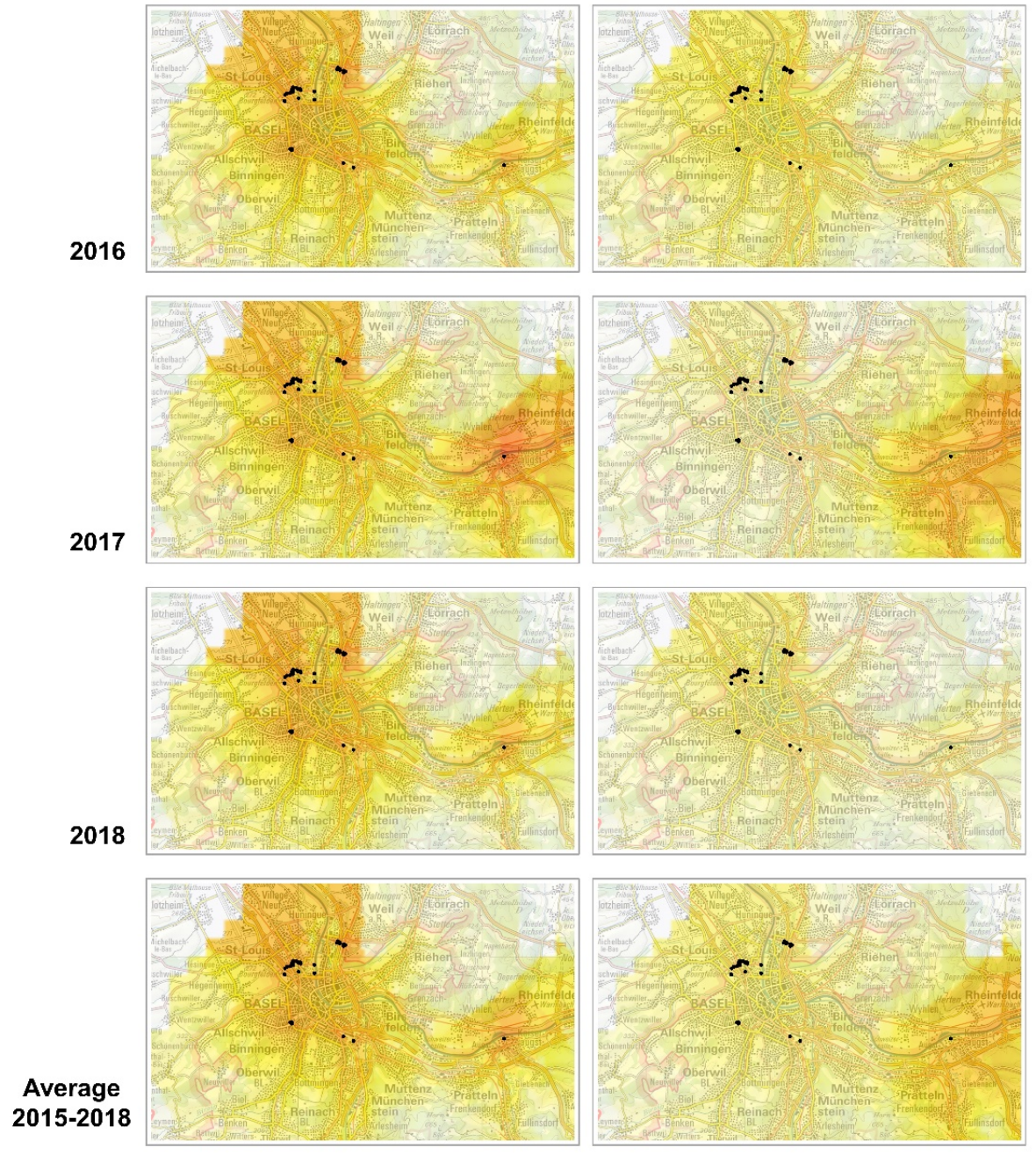

Figure 4. Maps of probabilities of establishment for Basel predicted by the regularized logistic regression for 2015 to 2018. The maps in the left column correspond to the risk estimates based on the meteorological conditions of each individual year (maps named 2015, 2016, 2017, 2018) and the average risk over the four years (map named average 2015-2018). Those in the right column represent the uncertainty of the corresponding predictions (higher values representing more uncertain predictions). The colour gradient shows the probability of establishment or the normalized stand- 
ard deviation from 0 (white) to 1 (red). Black dots show the positions where Ae. albopictus was established in 2019, which are used to compute the carD feature (distance from the closest cell with establishment in the previous year). Maps modified from https://map.geoadmin.ch/.

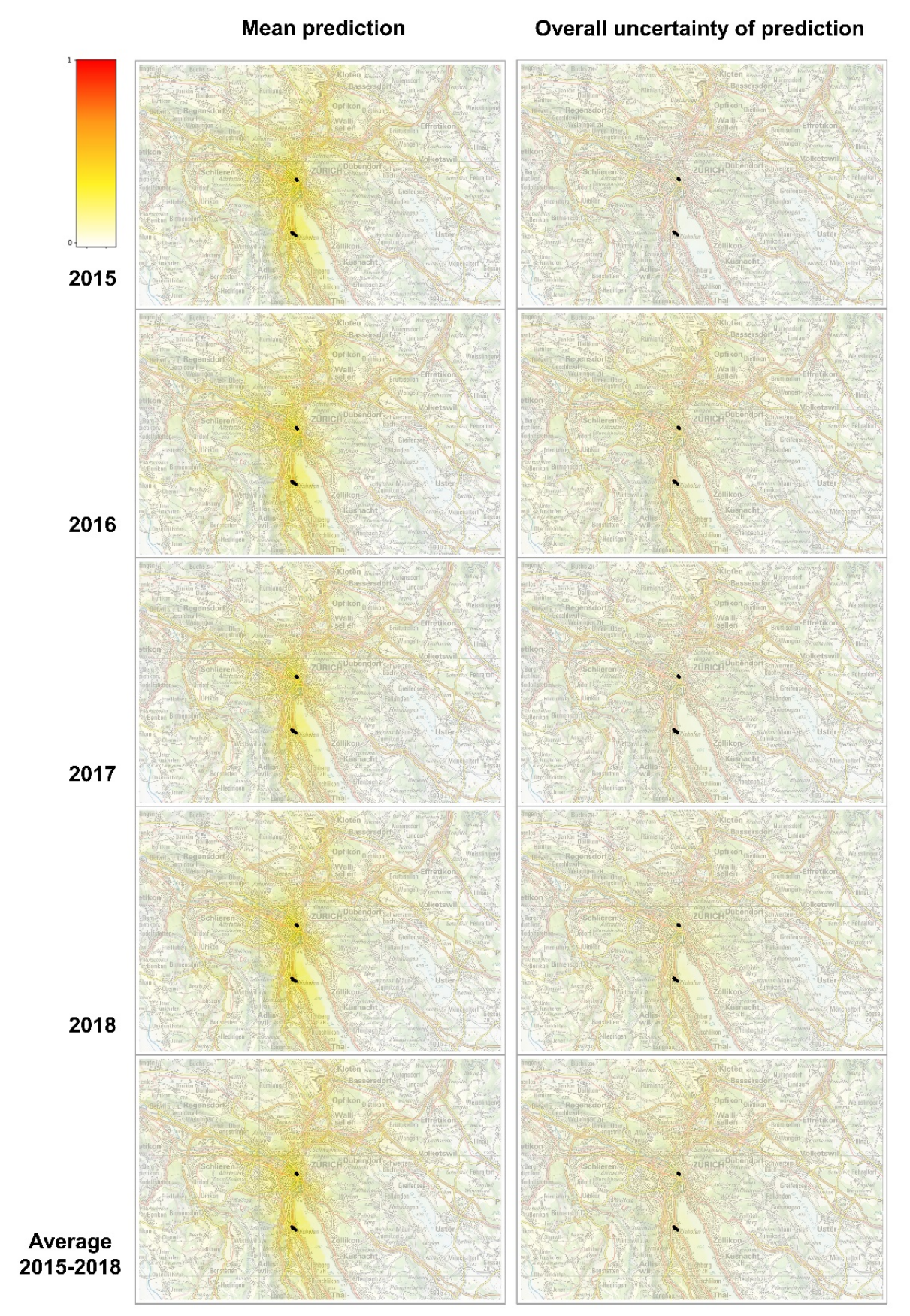

Figure 5. Maps of probabilities of establishment for Zurich predicted by the regularized logistic regression for 2015 to 2018. The maps in the left column correspond to the risk estimates based on the meteorological conditions of each individual year (maps named 2015, 2016, 2017, 2018) and the average risk over the four years (map named average 2015-2018). Those in the right column represent the uncertainty of the corresponding predictions (higher values representing more uncertain 
predictions). The colour gradient shows the probability of establishment or the normalized standard deviation from 0 (white) to 1 (red). Black dots show the positions where Ae. albopictus was established in 2019, which are used to compute the carD feature (distance from the closest cell with establishment in the previous year). Maps modified from https://map.geoadmin.ch/.

\section{Discussion}

We developed a regularized logistic regression that used the historical entomological Ae. albopictus data from ovitraps in Ticino from 2005 to 2012 as response variable in the training process. In the validation process, the probability of establishment of Ae.albopictus in Ticino was predicted both by cross-validation over the training years 2007 to 2012 and for the test years 2013, 2014 and 2015 and the entomological data for the same years were used to test the performance of the ensemble model separately for each year. The best performance was obtained in cross-validation on data from 2007 to 2012, with an AUC of 0.856 , whereas establishment was more difficult to predict for test years from 2013 to 2015 (AUCs between 0.67 and 0.75). We believe that this is due to the fact that, whereas in the initial phase of Ae. albopictus colonization the distance from already established colonies is a good predictor of new establishments, in more recent years where mosquitoes are well settled, establishment in specific areas is less related to the nearby situation and more affected by factors, such as possible treatments against the vector or specific features of the traps and traps' environment, that were not observed. However, since our interest is to predict the spread of mosquitoes in areas of Switzerland where they are not yet settled, an AUC of 0.856 on the years of mosquitoes' diffusion allows us to expect the probabilities of establishment predicted by the ensemble in unobserved cells to be quite informative about the most probable directions of Ae. albopictus further diffusion.

When building suitability maps of Ae. albopictus establishment, we used meteorological data from years 2015, 2016, 2017 and 2018 as scenarios for the meteorological conditions of the coming years. Temperature wise, these years are similar. They represent some of the warmest years in Switzerland since the first measurements in 1864, all of which have been recorded after 2010 [31]. Their average annual temperature exceeds the average of the years $1981-2010$ by 0.7 to $1.5^{\circ} \mathrm{C}$, with 2018 being the hottest year since the beginning of measurements in 1864 . These scenarios can also be considered representative of the most recent years 2019 and 2020 and probably also realistic for the coming decade. Indeed, the average annual temperatures of 2019 and 2020 follow the same trend, exceeding the average of the years $1981-2010$ by 1.1 and $1.5^{\circ} \mathrm{C}$, respectively. Differently, no change in average summer precipitation has been discerned in Switzerland so far. Nevertheless, there are clear signs that heavy precipitation pattern is slowly changing, with intensity and frequency of heavy rainfall increasing in all seasons and regions of Switzerland.

Of the 79 candidate predictors (including meteorological indicators, terrain morphology, type of land coverage, total human population and travel distance from the nearest established cell) contemplated as potentially relevant for predicting the probability of Ae. albopictus establishment in Ticino, only ten were considered informative by at least half of the eight ensemble models. Interpreting data-driven models can be hard and misleading, especially when features are correlated, as in this case. However, we hereafter analyse the parameters of the ensemble model and try to provide an interpretation for them with the aim of suggesting hypotheses that might stimulate the discussion and inspire further research.

Altitude, which is regarded as a proxy of temperature, contributed significantly to the prediction of establishment: the higher the elevation of a cell is, the lower the probability of Ae. albopictus establishment will be. The effect of altitude has been well observed in Ticino, where the establishment of the mosquito is delayed above 400 m.a.s.l. [14]. Out of 30 temperature predictors at a seasonal temporal scale and four temperature predictors at a two-week temporal scale, only four were considered informative. Two of them 
account for temperatures in the warm season: the fifth percentile of the minimum temperature in the warm season (wTmin_p5) shows a negative association with the probability of establishment, while the $75^{\text {th }}$ percentile of the maximum temperature in the warm season (wTmax_p75) showed a positive relation, the strongest effect being the one of wTmin_p5. The 5\% of smallest Tmin can be expected to refer to spring or autumn temperature, whereas the $75 \%$ of highest values of Tmax ca be expected to happen during the summer. The learned model is therefore saying that somehow colder spring or autumn season as well as hotter summers may favour Ae. albopictus establishment. The direction of wTmin_p5 association with the establishment goes in the opposite direction than the one we had expected, since high temperature are, in general, expected to increase the suitability of an area to Ae. albopictus establishment. However, the wTmin_p5 feature might indirectly account for the effect of other non-observed variables, e.g., humidity, higher values of wTmin_p5 being possibly associated to drier conditions in spring or autumn.

Other two selected predictors account for cold-season temperatures: the $25^{\text {th }}$ percentile of the maximum temperature in the cold season (cTmax_p25), which showed a positive relation to the probability of establishment, and the average minimum temperature of one of the coldest two-week periods of the year (minMavgTmin), which showed a negative relation but also contributed much less than cTmax_p25 to the probability of establishment. The model therefore describes a situation in which Ae. albopictus is more likely to establish in case of warmer winters, although, surprisingly, the probability of establishment is slightly reduced when the temperatures observed during protracted cold periods are higher. Perhaps, the lack of consensus in these two latter predictors is not surprising, since the response variable Established is based on the reproductive success in the warm season, more than on the overwintering success of diapausing eggs. Nonetheless, the pattern of invasion of Ae. albopictus in Ticino [13,14] suggests a connection between the establishment and overwintering: if the mosquitoes are established in an area, it is very likely that they will start to overwinter in the area and that they will be present the following years as well.

Precipitation can have both a positive effect on the larval carrying capacity of breeding sites and a negative effect on the mosquito flying activity and reproductive cycle interrupting it by washing away aquatic stages from container breeding sites $[45,46]$. This ambivalent effect was indeed detected by our model. The importance of the occurrence of rainfall was highlighted by the positive relation between the average rain observed during the least rainy two-week periods of the warm season (minMavgRAIN). However, too much rainfall might have a negative impact on the survival of aquatic stages or the abundance and distribution of mosquito breeding habitats, as indicated by the negative relation of the average of precipitations in the warm season (wRAIN_avg) with the probability of establishment. This could be due to different reasons such as the increase of breeding sites other than ovitraps (and therefore less recovery of eggs in the ovitraps), the decreased activity of females, or the larvae being washed away by intense precipitations. The latter option might increase in importance in the future with the expected increase in heavy rainfall events. The results also suggested some effect of rainfall in the cold season, with a positive impact of the $75^{\text {th }}$ percentile of the precipitations in this part of the year (cRAIN_75).

The presence and abundance of resident humans does not seem to play a role in the probability of establishment, but the feature indirectly related to humans' movement, carD, was selected as one of the key drivers of Ae. albopictus spread. This predictor measured the road-based distance in minutes of travel by car from the nearest cell established in the previous year and showed a significant negative relation to the probability of establishment: the higher the distance to nearest established cell is, the lower the probability of establishment will be, and vice versa. This is, to our knowledge, the first time that this variable is evaluated in a model on Ae. albopictus dispersal. Yet, the existence of passive dispersal of adult tiger mosquitoes in private vehicles at both local and 
medium-range scales has been confirmed in other settings [39] and its importance in Switzerland is supported by the findings of positive ovitraps along the major transportation routes $[13,15]$.

The percentage of industrial or commercial units covering a cell showed a negative relation to the probability of establishment of Ae. albopictus: the higher the percentage is, the lower the probability of establishment will be. In Ticino, as observed by Flacio et al. [14], industrial areas in several municipalities were apparently colonised by the mosquito before residential areas with a lead time of two to three years. Indeed, industrial areas may function as key ports (transport of goods and working places of commuters from areas where the mosquito has already established) of passive mosquito introductions. However, later on, the mosquito was more present in the residential than in industrial areas, since, perhaps, more breeding sites are available in residential areas.

The validated model was used to extrapolate the probability of establishment on the whole Swiss territory with promising results. The forecasts are similar among the different years analysed. In 2016, the areas at risk of establishment are the same as in the map of mean prediction 2015-2018 but the risk is accentuated, indicating that this year seemed to be more favourable to the establishment of Ae. albopictus, and the uncertainty of the ensemble model estimates was low. The areas identified by the model as suitable for Ae. albopictus establishment are consistent with the actual findings of ovitraps positive for tiger mosquito. The maps show high probability of establishment along the Central Plateau, a geographical region that stretches from Lake Geneva in the southwest to Lake Constance in the northeast. This region is characterized by a flat to hilly landscape (with an average altitude between 400 and 700 m.a.s.1.), compared to the other more mountainous regions of Switzerland, and is also the most densely populated region of Switzerland. Correspondingly, positive ovitraps have been observed, from southwest to northeast of the Central Plateau, in the cantons of Geneva, Vaud, Fribourg, Solothurn, Luzern, Aargau, Zurich and Schaffhausen (Swiss Mosquito Network and info fauna Swiss Centre for Cartography of the Fauna). High risk of establishment has also been identified in the northern part of canton Basel-Landt, where positive ovitraps have been recorded, and in the canton Basel-Stadt, where an established colony and several foci are present in the city of Basel. At the same time, a high risk of establishment is predicted in the lower Rhone Valley in the Canton of Valais. Interestingly, the high degree of risk predicted in Geneva and in the lower valleys of Valais, although with a certain degree of incertitude among the models, is very similar in colours to the degree of risk predicted in Ticino, where the tiger mosquito is already established and where the ensemble model was validated with and AUC of 0.85. Mosquito-wise, the main difference between Geneva/Valais and Ticino is that in Geneva/Valais the introduction pressure from adjacent established areas is much lower at present. Meanwhile, in the nearby France, Ae. albopictus is expanding northwards [47] and might soon become a source of mosquitoes for these Swiss regions. Thus, our results suggest that authorities in the high-risk areas should be alerted and surveillance should be increased.

We compared our predictions with those of a previous prediction for invasion of $A e$. albopictus in Switzerland produced in 2013 with a different approach. The previous model did not use entomological data but instead suitable areas for adult survival and overwintering of diapausing eggs were calculated analysing remotely sensed land surface temperature data recorded by the MODIS satellite sensors based on set climatic thresholds [22]. The predictions of suitability for adults for 2011 and 2035, which were based on a climate change scenario, are very similar to our predictions for establishment, which were obtained using historical tiger mosquito data instead of climatic thresholds.

The model was also used to extrapolate the probability of establishment in the cities of Basel and Zurich, where only a small number of occurrences is available for analysis. For all years analysed, Basel seems to be at higher risk of establishment than Zurich, although the uncertainty of prediction (standard deviation of the models) is in general higher in Basel than in Zurich. In 2016, a year considered favourable for the establish- 
ment of Ae. albopictus in Switzerland, the probability of establishment for Basel is similar to the other years, while it appears lower for Zurich. Overall, we can conclude that the city of Basel might need to intensify the surveillance for Ae. albopictus foci of introduction, perhaps adopting a grid system to standardize the distribution of ovitraps on the territory, as it has been done in Ticino [13]. The establishment in the city of Zurich seems less probable, although the present model did not consider yet the potentially positive effect of microhabitats such as catch basins on the probability of overwintering of diapausing eggs [48]. Thus, it appears that the surveillance of Ae. albopictus in Zurich could still focus on the hot spots of introduction, such as the international bus station in the city centre and the Wollishofen neighbourhood where tiger mosquito adults were observed. In 2021, after three years of surveillance and control including reduction of breeding sites, larvicide treatment of catch basins, information of the residents and door-to-door visits, ovitraps were no longer positive for Ae. albopictus in the Wollishofen neighbourhood [49]. There is a high likelihood that Ae. albopictus was successfully eradicated in this area, which is a very rewarding and encouraging outcome for authorities and scientists.

\section{Conclusions}

We developed a model that learns from the historical occurrence data of Ae. albopictus in Ticino, Switzerland, acquired through years of surveillance, and predicts the probability of establishment of the mosquito on the area. The model can be used to extrapolate the probability of establishment outside the training area (i.e., whole Switzerland), where the mosquito is not yet established, with promising results. We can see from the predictions that various parts of Switzerland are at risk for establishment of Ae. albopictus. Additionally, the model can also be used to create scenarios of establishment and spread in smaller specific areas where there are already localized colonies, as in the city of Basel, or small foci of introduction, as in the city of Zurich. The risk maps with the probability of establishment of Ae. albopictus can help cantonal and municipal authorities in optimizing efforts and resources by targeting vector surveillance and control in the areas with higher risk of establishment.

Supplementary Materials: The following supporting information can be downloaded at: www.mdpi.com/xxx/s1, Table S1: Candidate predictors used for modelling Ae. albopictus habitat suitability.

Author Contributions: Conceptualization, D.R., F.M., D.H., L.E., L.A., V.G., M.T. and E.F.; methodology, D.R., L.E., F.M., D.H. and E.F.; software, F.M., D.H., N.V. and G.D.R.; validation, F.M. and D.H.; formal analysis, F.M., D.H. and L.A.; investigation, F.M., D.H., L.A., L.E., E.F.; resources, D.R., F.M., D.H., N.V., L.E., M.T. and E.F.; data curation, F.M., D.H. and L.E. ; writing-original draft preparation, D.R. and F.M.; writing - review and editing, D.R., F.M., D.H., V.G., L.A. and E.F.; visualization, D.R., F.M. and D.H.; supervision, D.R.; project administration, D.R.; funding acquisition, D.R., F.M., M.T. and E.F. All authors have read and agreed to the published version of the manuscript.

Funding: This research was funded by the University of Applied Sciences and Arts of Southern Switzerland through an internal fund; the Swiss Federal Office for the Environment and the Swiss Confederation as part of Pilot programme 'Adaptation to climate change'.

Institutional Review Board Statement: Not applicable

Informed Consent Statement: Not applicable.

Data Availability Statement: Data was obtained from the different sources cited in the text and is available with the permission of these sources.

Acknowledgments: We are very grateful to the Swiss Mosquito Network, in particular to Susanne Biebinger, Daniel Cherix, Gabi Müller, Pie Müller and Barbara Wiesendanger, along with info fauna - Swiss Centre for Cartography of the Fauna, for providing occurrence data on Ae. albopictus and sharing their knowledge. A big thank you also to Basil Gerber from the Swiss Federal Office for the Environment for his constant support and practical application of the tools obtained. We thank 
all the municipalities and authorities involved in the surveillance and control of the tiger mosquito. We also thank the many people who collected and analyzed the samples and Mabritec AG for the analyses with MALDI-TOF mass spectrometry. Many thanks to Andrea Danani (IDSIA-SUPSI) and Alessio Spataro (IST-SUPSI) for their collaboration. Data for the model were also provided by MeteoSwiss, the Swiss Federal Office of Meteorology and Climatology, the European Environment Agency, the Swiss Federal Office of Topography and the Swiss Federal Statistical Office. Map data copyrighted OpenStreetMap contributors and available from https://www.openstreetmap.org.

Conflicts of Interest: The authors declare no conflict of interest.

\section{Appendix A}

In order to account for meteorological conditions on a more refined temporal scale, we also included some features based on two-week statistics of MeteoSwiss data series. These features also account better for prolonged extreme meteorological conditions than seasonal predictors. For this, we computed the moving averages (Mavg) of the Tmin, Tmax and RAIN series, the moving maximum (Mmax) of the Tmin series and the moving minimum (Mmin) of the Tmax series over time windows of two weeks from November of year $y-1$ to October of year y for the temperature series and from May to September (warm season) for the RAIN series. This way, we obtained 5 new time series (MavgTmin, MavgTmax, MavgRAIN, MmaxTmin and MminTmax) showing for each day of the considered periods the average, the minimum or the maximum values of the previous 15 days. From those time series, we derived the following six features:

- The minimum value of the MmaxTmin and of the MavgTmin series. The former, named minMmaxTmin is associated to the two-week period during which the daily values of Tmin have had the smallest upper bound of the year, since Tmin have been constantly below minMmaxTmin; the latter, named minMavgTmin corresponds to the minimum value reached during the year by the biweekly average of Tmin. They both are meant to represent how cold some of the coldest two-week periods of the year were, minMmaxTmin focusing on the two weeks with the lowest upper bound of Tmin and minMavgTmin on the two weeks with the smallest average values of Tmin.

- Similarly, we have computed the maximum value of the Mmin and of the Mavg of Tmax. The former, named maxMminTmax is associated to the two-week period during which the daily values of Tmax have had the largest lower bound of the year, since Tmax have been constantly above maxMminTmax; the latter, named maxMavgTmax corresponds to the maximum value reached during the year by the biweekly average of Tmax. They both are meant to represent how hot some of the hottest two-week periods of the year were, maxMminTmax focusing on the two weeks with the largest lower bound of Tmax and maxMavgTmax on the two weeks with the largest average values of Tmax.

- Finally, we have retained the maximum and minimum of MavgRAIN during the warm season (named, maxMavgRAIN and minMavgRAIN), which are meant to represent, respectively, the average rain observed during one of the most and one of the least rainy two-week periods of the warm season, that is, those with the average value of precipitation, respectively, highest and lowest.

\section{Appendix B}

We analysed the contribution of each selected feature in each prediction from the eight individual models over years 2005 to 2015. The contribution is computed as the product of the feature coefficient and the normalized value of the feature. Figure A1 shows the boxplots of the distance of each feature contribution from its average. We see that the greatest contributions are due to car distances (since big distances strongly re- 
duce establishment suitability). Relevant contributions are also imputable to cTmax_p25, wRAIN_avg, wTmin_p5 and elevation. Very small contribution, instead, are those of clc121, minMavgRAIN and wTmax_p75, minMavgTmin and cRAIN_p75, except for a small number of outliers.

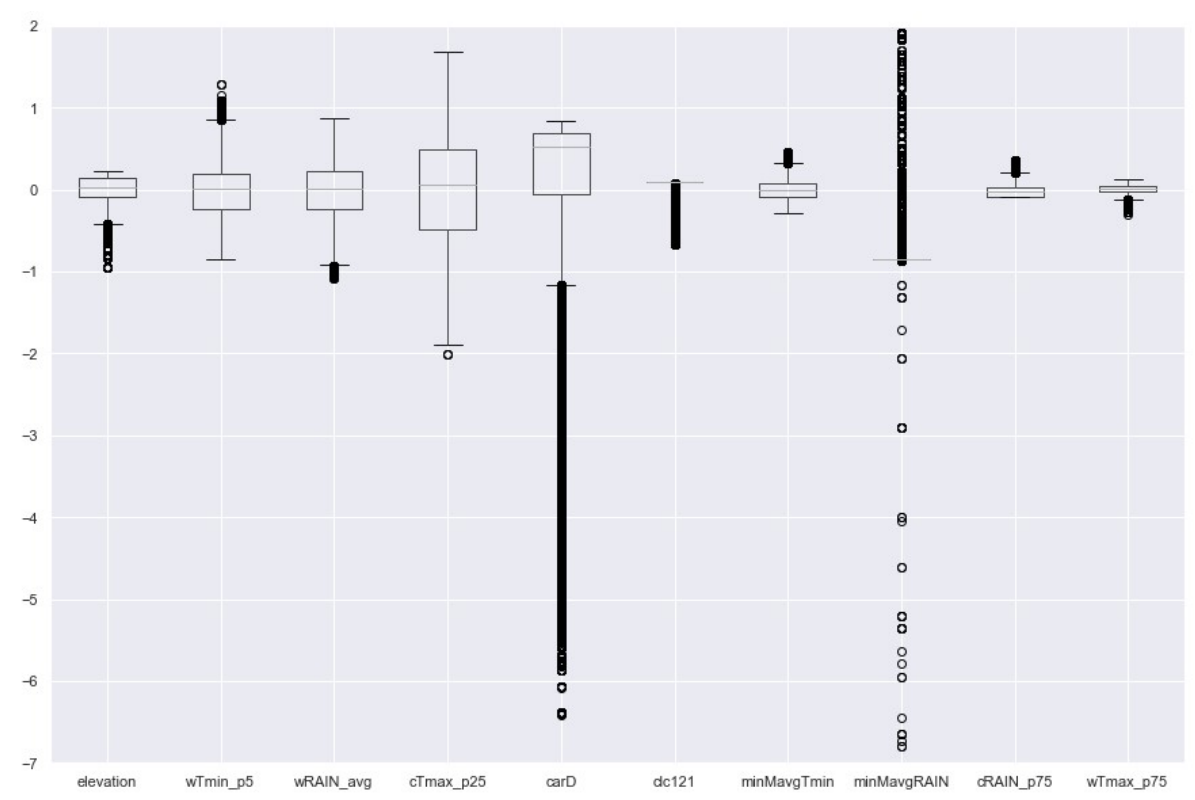

Figure A1. Contribution of the ten most selected features.

\section{References}

1. Paupy, C.; Delatte, H.; Bagny, L.; Corbel, V.; Fontenille, D. Aedes albopictus, an arbovirus vector: from the darkness to the light. Microbes Infect. 2009, 11, 1177-1185, doi:10.1016/j.micinf.2009.05.005.

2. Medlock, J.M.; Hansford, K.M.; Versteirt, V.; Cull, B.; Kampen, H.; Fontenille, D.; Hendrickx, G.; Zeller, H.; Van Bortel, W.; Schaffner, F. An entomological review of invasive mosquitoes in Europe. Bull. Entomol. Res. 2015, 105, 637-663, doi:10.1017/S0007485315000103.

3. Cunze, S.; Koch, L.K.; Kochmann, J.; Klimpel, S. Aedes albopictus and Aedes japonicus - two invasive mosquito species with different temperature niches in Europe. Parasit. Vectors 2016, 9, 1-12, doi:10.1186/s13071-016-1853-2.

4. Cunze, S.; Kochmann, J.; Koch, L.K.; Klimpel, S. Aedes albopictus and its environmental limits in Europe. PLoS One 2016, 11, 1-14, doi:10.1371/journal.pone.0162116.

5. Armbruster, P.A. Photoperiodic diapause and the establishment of Aedes albopictus (Diptera: Culicidae) in North America. J. Med. Entomol. 2016, 53, 1013-1023, doi:10.1093/jme/tjw037.

6. Pluskota, B.; Jöst, A.; Augsten, X.; Stelzner, L.; Ferstl, I.; Becker, N. Successful overwintering of Aedes albopictus in Germany. Parasitol. Res. 2016, 115, 3245-3247, doi:10.1007/s00436-016-5078-2.

7. Medlock, J.M.; Hansford, K.M.; Schaffner, F.; Versteirt, V.; Hendrickx, G.; Zeller, H.; Van Bortel, W. A review of the invasive mosquitoes in Europe: Ecology, public health risks, and control options. Vector Borne Zoonotic Dis. 2012, 12, 435-447, doi:10.1089/vbz.2011.0814.

8. Bonizzoni, M.; Gasperi, G.; Chen, X.; James, A.A. The invasive mosquito species Aedes albopictus: current knowledge and future perspectives. Trends Parasitol. 2013, 29, 460-468.

9. Barzon, L. Ongoing and emerging arbovirus threats in Europe. J. Clin. Virol. 2018, 107, 38-47. 
10. Messina, J.P.; Brady, O.J.; Golding, N.; Kraemer, M.U.G.; Wint, G.R.W.; Ray, S.E.; Pigott, D.M.; Shearer, F.M.; Johnson, K.; Earl, L.; et al. The current and future global distribution and population at risk of dengue. Nat. Microbiol. 2019, 4, 1508-1515, doi:10.1038/s41564-019-0476-8.

11. Flacio, E.; Lüthy, P.; Patocchi, N.; Guidotti, F.; Tonolla, M.; Peduzzi, R. Primo ritrovamento di Aedes albopictus in Svizzera. Boll. della Soc. Ticin. di Sci. Nat. 2004, 92, 141-142.

12. Wymann, M.N.; Flacio, E.; Radczuweit, S.; Patocchi, N.; Lüthy, P. Asian tiger mosquito (Aedes albopictus) - a threat for Switzerland? Euro Surveill. 2008, 13, 8058, doi:10.2807/ese.13.10.08058-en.

13. Flacio, E.; Engeler, L.; Tonolla, M.; Lüthy, P.; Patocchi, N. Strategies of a thirteen year surveillance programme on Aedes albopictus (Stemomyia albopicta) in southern Switzerland. Parasit. Vectors 2015, 8, 208-225.

14. Flacio, E.; Engeler, L.; Tonolla, M.; Müller, P. Spread and establishment of Aedes albopictus in southern Switzerland between 2003 and 2014: an analysis of oviposition data and weather conditions. Parasit. Vectors 2016, 9, 304-317, doi:10.1186/s13071-016-1577-3.

15. Müller, P.; Engeler, L.; Vavassori, L.; Suter, T.; Guidi, V.; Gschwind, M.; Tonolla, M.; Flacio, E. Surveillance of invasive Aedes mosquitoes along Swiss traffic axes reveals different dispersal modes for Aedes albopictus and Ae. japonicus. PLoS Negl. Trop. Dis. 2020, 14, 1-20, doi:10.1371/journal.pntd.0008705.

16. Biebinger, S. Asiatische Tigermücke. Überwachung und Bekämpfung im Kanton Basel-Stadt 2020; Report of Gesundheitsdepartement des Kantons Basel-Stadt, Kantonales Laboratorium 2020; https://www.kantonslabor.bs.ch/umwelt/neobiota/tigermuecke.html;

17. Peterson, A.T.; Soberón, J. Species distribution modeling and ecological niche modeling: Getting the concepts right. Nat. Conserv. 2012, 10, 102-107, doi:10.4322/natcon.2012.019.

18. Melo-Merino, S.M.; Reyes-Bonilla, H.; Lira-Noriega, A. Ecological niche models and species distribution models in marine environments: A literature review and spatial analysis of evidence. Ecol. Modell. 2020, 415, doi:10.1016/j.ecolmodel.2019.108837.

19. Delatte, H.; Gimonneau, G.; Triboire, A.; Fontenille, D. Influence of temperature on immature development, survival, longevity, fecundity, and gonotrophic cycles of Aedes albopictus, vector of chikungunya and dengue in the Indian Ocean. J. Med. Entomol. 2009, 46, 33-41, doi:10.1603/033.046.0105.

20. Roiz, D.; Rosà, R.; Arnoldi, D.; Rizzoli, A. Effects of temperature and rainfall on the activity and dynamics of host-seeking Aedes albopictus females in northern Italy. Vector Borne Zoonotic Dis. 2010, 10, 811-816, doi:10.1089/vbz.2009.0098.

21. Fischer, D.; Thomas, S.M.; Neteler, M.; Tjaden, N.B.; Beierkuhnlein, C. Climatic suitability of Aedes albopictus in Europe referring to climate change projections: Comparison of mechanistic and correlative niche modelling approaches. Euro Surveill. 2014, 19, doi:10.2807/1560-7917.ES2014.19.6.20696.

22. Neteler, M.; Metz, M.; Rocchini, D.; Rizzoli, A.; Flacio, E.; Engeler, L.; Guidi, V.; Lüthy, P.; Tonolla, M. Is Switzerland suitable for the invasion of Aedes albopictus? PLoS One 2013, 8, 1-10, doi:10.1371/journal.pone.0082090.

23. European Centre for Disease Prevention and Control (ECDC) Environmental risk mapping : Aedes albopictus in Europe; Stockholm: ECDC, 2013;

24. Manica, M.; Filipponi, F.; D’Alessandro, A.; Screti, A.; Neteler, M.; Rosà, R.; Solimini, A.; della Torre, A.; Caputo, B. Spatial and temporal hot spots of Aedes albopictus abundance inside and outside a South European metropolitan area. PLoS Negl. Trop. Dis. 2016, 10, 1-17, doi:10.1371/journal.pntd.0004758.

25. Richman, R.; Diallo, D.; Diallo, M.; Sall, A.A.; Faye, O.; Diagne, C.T.; Dia, I.; Weaver, S.C.; Hanley, K.A.; Buenemann, M. Ecological niche modeling of Aedes mosquito vectors of chikungunya virus in southeastern Senegal. Parasit. Vectors 2018, 11, 1-17, doi:10.1186/s13071-018-2832-6.

26. Scavuzzo, J.M.; Trucco, F.; Espinosa, M.; Tauro, C.B.; Abril, M.; Scavuzzo, C.M.; Frery, A.C. Modeling Dengue vector 
population using remotely sensed data and machine learning. Acta Trop. 2018, 185, 167-175,

doi:10.1016/j.actatropica.2018.05.003.

27. Cianci, D.; Hartemink, N.; Ibáñez-Justicia, A. Modelling the potential spatial distribution of mosquito species using three different techniques. Int. J. Health Geogr. 2015, 14, 1-10, doi:10.1186/s12942-015-0001-0.

28. Früh, L.; Kampen, H.; Kerkow, A.; Schaub, G.A.; Walther, D.; Wieland, R. Modelling the potential distribution of an invasive mosquito species: comparative evaluation of four machine learning methods and their combinations. Ecol. Modell. 2018, 388, 136-144, doi:10.1016/j.ecolmodel.2018.08.011.

29. Ding, F.; Fu, J.; Jiang, D.; Hao, M.; Lin, G. Mapping the spatial distribution of Aedes aegypti and Aedes albopictus. Acta Trop. 2018, 178, 155-162, doi:10.1016/j.actatropica.2017.11.020.

30. Kraemer, M.U.G.; Sinka, M.E.; Duda, K.A.; Mylne, A.Q.N.; Shearer, F.M.; Barker, C.M.; Moore, C.G.; Carvalho, R.G.; Coelho, G.E.; Van Bortel, W.; et al. The global distribution of the arbovirus vectors Aedes aegypti and Ae. albopictus. Elife 2015, 4, 1-18, doi:10.7554/eLife.08347.

31. Federal Office for the Environment (FOEN); Federal Office of Meteorology and Climatology (MeteoSwiss); National Center for Climate Services (NCCS) Management summary: Climate change in Switzerland. Indicators of driving forces, impact and response.; State of the environment no. 2013: 11 pp., 2020;

32. Swisstopo DHM25 / 200 The digital height model of Switzerland with a 200m grid Available online: https://shop.swisstopo.admin.ch/en/products/height_models/dhm25200 (accessed on Oct 18, 2021).

33. European Centre for Disease Prevention and Control (ECDC) Guidelines for the surveillance of invasive mosquitoes in Europe; Stockholm: ECDC, 2012;

34. Federal Office of Meteorology and Climatology (MeteoSwiss) SwissMetNet: Ein Messnetz für die Zukunft; Federal Office of Meteorology and Climatology, Zürich, 2010;

35. Frei, C. Interpolation of temperature in a mountainous region using nonlinear profiles and non-Euclidean distances. Int. J. Climatol. 2014, 34, 1585-1605, doi:10.1002/joc.3786.

36. Frei, C.; Schöll, R.; Fukutome, S.; Schmidli, J.; Vidale, P.L. Future change of precipitation extremes in Europe: Intercomparison of scenarios from regional climate models. J. Geophys. Res. 2006, 111, doi:10.1029/2005JD005965.

37. Zaharia, M.; Xin, R.S.; Wendell, P.; Das, T.; Armbrust, M.; Dave, A.; Meng, X.; Rosen, J.; Venkataraman, S.; Franklin, M.J.; et al. Apache Spark: A unified engine for big data processing. Commun. ACM 2016, 59, 56-65, doi:10.1145/2934664.

38. Steinmeier, C. CORINE Land Cover 2012 Switzerland. Final Report [published online 2014]; Available from https://www.wsl.ch;

39. Eritja, R.; Palmer, J.R.B.; Roiz, D.; Sanpera-Calbet, I.; Bartumeus, F. Direct evidence of adult Aedes albopictus dispersal by car. Sci. Rep. 2017, 7, 1-15, doi:10.1038/s41598-017-12652-5.

40. Luxen, D.; Vetter, C. Real-time routing with OpenStreetMap data. In Proceedings of the 19th ACM SIGSPATIAL International Conference on Advances in Geographic Information Systems GIS '11; Chicago, Illinois, 2011; pp. 513-516.

41. OpenStreetMap contributors 2017 Planet dump Available online: https://planet.openstreetmap.org (accessed on Jun 1, 2021).

42. Rokach, L. Ensemble-based classifiers. Artif. Intell. Rev. 2010, 33, 1-39, doi:10.1007/s10462-009-9124-7.

43. Pedregosa, F.; Varoquaux, G.; Gramfort, A.; Michel, V.; Thirion, B.; Grisel, O.; Blondel, M.; Prettenhofer, P.; Weiss, R.; Dubourg, V.; et al. Scikit-learn: Machine Learning in Python. J. Mach. Learn. Res. 2011, 12, 2825-2830.

44. Tibshirani, R. Regression Shrinkage and Selection via the Lasso. J. R. Stat. Soc. Ser. B 1996, 58, 267-288, doi:10.1111/j.2517-6161.1996.tb02080.x.

45. Dieng, H.; Rahman, G.M.S.; Hassan, A.A.; Salmah, M.R.C.; Satho, T.; Miake, F.; Boots, M.; Sazaly, A. The effects of simulated rainfall on immature population dynamics of Aedes albopictus and female oviposition. Int. J. Biometeorol. 2012, 56, 113-120, doi:10.1007/s00484-011-0402-0.

46. Dickens, B.L.; Sun, H.; Jit, M.; Cook, A.R.; Carrasco, L.R. Determining environmental and anthropogenic factors which 
explain the global distribution of Aedes aegypti and Ae. albopictus. BMJ Glob. Heal. 2018, 3, 1-11, doi:10.1136/bmjgh-2018-000801.

47. Roques, L.; Boivin, T.; Papaïx, J.; Soubeyrand, S.; Bonnefon, O. Dynamics of Aedes albopictus invasion. Insights from a spatio-temporal model. bioRxiv [Preprint] Available from https://doi.org/10.1101/2021.09.24.

48. Ravasi, D.; Guidi, V.; Flacio, E.; Lüthy, P.; Perron, K.; Lüdin, S.; Tonolla, M. Investigation of temperature conditions in Swiss urban and suburban microhabitats for the overwintering suitability of diapausing Aedes albopictus eggs. Parasit. Vectors 2018, 11, 1-9, doi:10.1186/s13071-018-2803-y.

49. Müller, G. Measures taken to eradicate a population of Aedes albopictus in a residential area in the City of Zurich. In Proceedings of the Swiss Vector Entomology Group (SVEG) Meeting 2021; Mendrisio, Switzerland, 2021. 